\title{
Automatic Calibration of 3D Ultrasound Probes
}

\author{
Thomas Lange $^{1}$, Silvan Kraft ${ }^{1}$, Sebastian Eulenstein ${ }^{1}$,Hans Lamecker ${ }^{2}$, \\ Peter M. Schlag 3 \\ ${ }^{1}$ Experimental and Clinical Research Center, Charité - Universitätsmedizin Berlin \\ ${ }^{2}$ Visualization and Data Analysis, Zuse Institute Berlin (ZIB) \\ ${ }^{3}$ Charite Comprehensive Cancer Center, Charité - Universitätsmedizin Berlin \\ thomas.lange@charite.de
}

\begin{abstract}
Navigation systems based on intra-operative ultrasound have been introduced for different surgical procedures and interventions. The accuracy of the ultrasound probe calibration is a main contribution to the overall accuracy of the navigation system. Different calibration methods based on phantoms have been introduced. The challenge of the calibration procedure is to identify the phantom structures accurately and possibly automatically in ultrasound images. In the majority of cases $2 \mathrm{D}$ ultrasound probes have been calibrated. The advantage of $3 \mathrm{D}$ probes is the acquisition of $3 \mathrm{D}$ ultrasound volumes out of the box enabling fast calibration approaches based on only one image acquisition. We introduce a new inexpensive and easily to manufacture planes phantom and automatic calibration algorithm for 3D ultrasound probes.
\end{abstract}

\section{Introduction}

Ultrasound is a relatively cheap and easy to integrate intra-operative imaging modality. Navigation systems based on intra-operative 3D ultrasound are in clinical use [1,2]. Different 3D ultrasound technologies exist [3] which can be divided into two groups: based on 2D or 3D ultrasound probes. Freehand 3D ultrasound is based on conventional 2D probes containing a $1 \mathrm{D}$ transducer array. A position sensor is attached to the $2 \mathrm{D}$ probe such that a $3 \mathrm{D}$ ultrasound volume can be compounded from a set of acquired image planes and their measured position and orientation. 3D probes either contain a $2 \mathrm{D}$ transducer array to directly measure a $3 \mathrm{D}$ volume or contain a $1 \mathrm{D}$ transducer array, which is mechanically or electronically swept over the image volume. 2D [1] as well as 3D [2] probes are used in 3D ultrasound navigation systems (Fig. 1a). A key element of such navigation systems is the spatial calibration of a position sensor attached to the ultrasound probe (Fig.1b). It is essential to know the spatial position and orientation of the ultrasound image coordinate system during acquisition. Spatial calibration is the procedure to determine a rigid transformation $T_{I \leftarrow S}$ from the position sensor $(S)$ attached to the ultrasound probe to this image coordinate system $(I)$. A point $x \in \mathbb{R}^{3}$ in the sensor coordinate system is transformed into its corresponding point $y \in \mathbb{R}^{3}$ in the image coordinate system by $y=T_{I \leftarrow S} x$.

Many different methods for ultrasound calibration have been developed. Nice reviews are given in $[4,5]$. In the majority of cases $2 \mathrm{D}$ ultrasound probes have 
been calibrated to enable freehand 3D ultrasound. Only rare literature about calibration of $3 \mathrm{D}$ probes exist. In principle the calibration methods for $2 \mathrm{D}$ probes can also be used for the default plane of a 3D probe, but much more information can be used from a 3D ultrasound volume compared to a $2 \mathrm{D}$ image plane. For the calibration of 2D probes usually several different image planes have to be acquired which image an invariant geometrical feature of a phantom from different directions. Then a set of transformation equations is constructed that match the known and identified phantom features and the equations are solved with respect to the transformation parameters. Typical phantoms contain crossing nylon wires to define points or membranes to define a plane which appears as a line in a $2 \mathrm{D}$ ultrasound image $[4,5]$. The biggest challenge in the calibration process is the accurate identification of the phantom features in the ultrasound images which often involves tedious and time-consuming manual interactions.

With 3D probes whole 3D features can be imaged in one image acquisition. In [6] a commercially available phantom with two egg-shaped features and in [7] an aluminum cube has been used as 3D feature. Here we introduce a similar approach using a very simple and inexpensive planes phantom and an automatic calibration procedure.

\section{Materials and Methods}

The main idea of the calibration approach is to measure or compute all other rigid transformations shown in Fig. 2a to determine the wanted calibration transformation $T_{I \leftarrow S}$. The position and orientation $T_{P \leftarrow W}$ of the phantom $(P)$ is measured by means of the tracking spheres on the phantom in the world coordinate system $(W)$ of the tracking camera. The position and orientation of the position sensor $(S)$ on the ultrasound probe is given by $T_{S \leftarrow W}$. If the position and orientation $T_{I \leftarrow P}$ of the phantom is determined in the ultrasound image

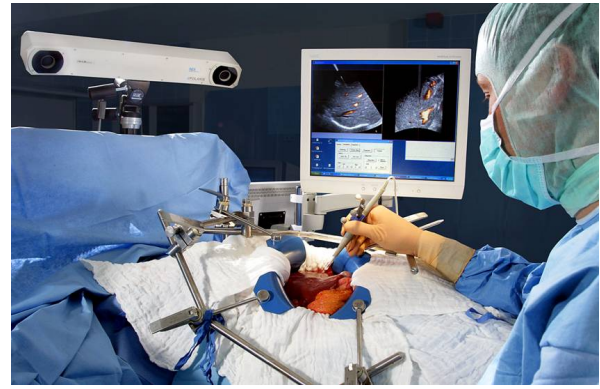

(a)

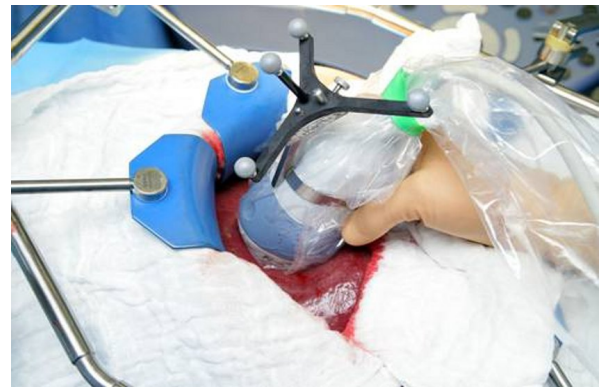

(b)

Fig. 1. a) Navigation system based on intra-operative 3D ultrasound for liver surgery. b) Optical position sensor attached to a $3 \mathrm{D}$ ultrasound probe applied directly onto the liver. 
coordinate system the calibration transformation can be computed by

$$
T_{I \leftarrow S}=T_{S \leftarrow W}^{-1} \cdot T_{P \leftarrow W} \cdot T_{I \leftarrow P}^{-1}
$$

\subsection{Planes Phantom}

The requirements to the calibration phantom are clear and easily detectable non-symmetric structures in ultrasound images without imaging artifacts by reverberations. In addition the phantom should be easily producible. We choose a simple water tank made from acrylic glass (Plexiglas). Water is the simplest medium to ensure the propagation of the ultrasound waves and the phantom features are still reachable with a tracked pointer in contrast to closed phantoms like in [6]. Non-elastic (hard) materials like the aluminum cube of Poon et al. [7] or the Plexiglas water tank lead to multiple reflexions and signal cancellation beneath the surface of the material. Therefore we used an elastic silicone to define the phantom features and filled the bottom of the Plexiglas tank with this material to prevent reverberations from the bottom and the sides of the tank. The silicone (Fegura Sil hydro spezial II, Feguramed GmbH, Germany) is used in dental technology to manufacture duplicates.

The calibration feature of the phantom is a non-symmetric configuration of four planes. One plane is parallel to the bottom of the water tank and the other three planes are angulated with approximately 12 degrees to the first plane.

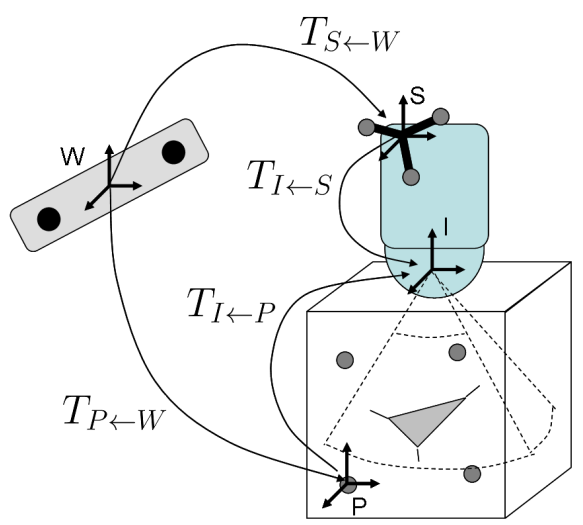

(a)

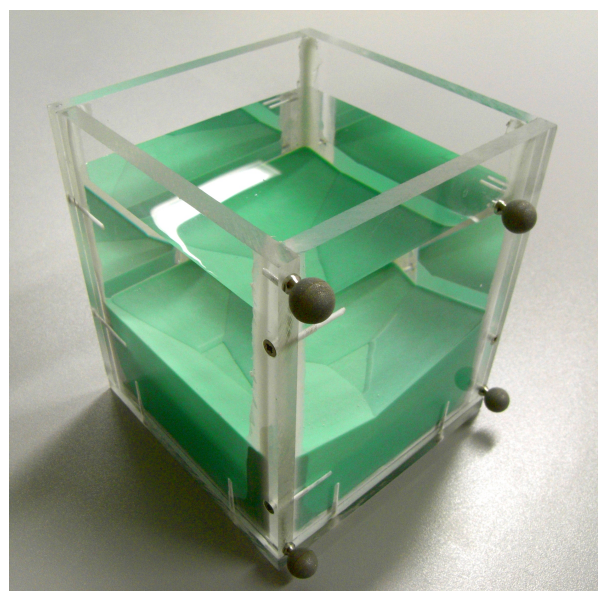

(b)

Fig. 2. a) Different coordinate systems and rigid transformations used in the calibration procedure. b) Water tank phantom with silicone planes features and optical tracking spheres. 


\subsection{Automatic Planes Detection}

For the determination of the transformation $T_{I \leftarrow P}$ we registered rigidly a virtual model of the phantom planes (Fig. 3a) to the image of the planes in the acquired ultrasound volume. In each iteration of the registration process linear profiles perpendicular to the plane model are evaluated in the ultrasound volume. If along a profile the intensity increases to a value above a given threshold a displacement vector between the current point on the model surface to the found point on the intensity profile is determined. For the set of displacement vectors an optimal rigid transformation is computed and applied to the current position and orientation of the model surface. This procedure is iterated until no further significant improvements can be reached.

\section{Results}

We evaluated the calibration procedure with the optical tracking system Polaris (NDI) and a GE Voluson 730 ultrasound machine equipped with a RAB2-5 abdominal 3D probe.

\subsection{Reconstruction of Phantom Geometry}

As the exact position and orientation of the planes in the phantom are not known by construction we measured them with the tracking system. For each of the four planes a set of 300 points on the surface of a plane was measured with a tracked pointer. A virtual plane was fitted to each of the point sets by minimizing the sum of squared differences between the point positions and the plane. The mean of the residual distances was for all four planes below $0.1 \mathrm{~mm}$ and the maximum below $0.4 \mathrm{~mm}$.

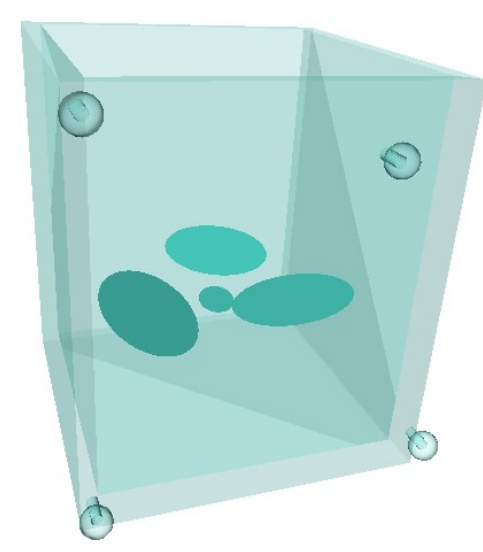

(a) virtual 3D planes model

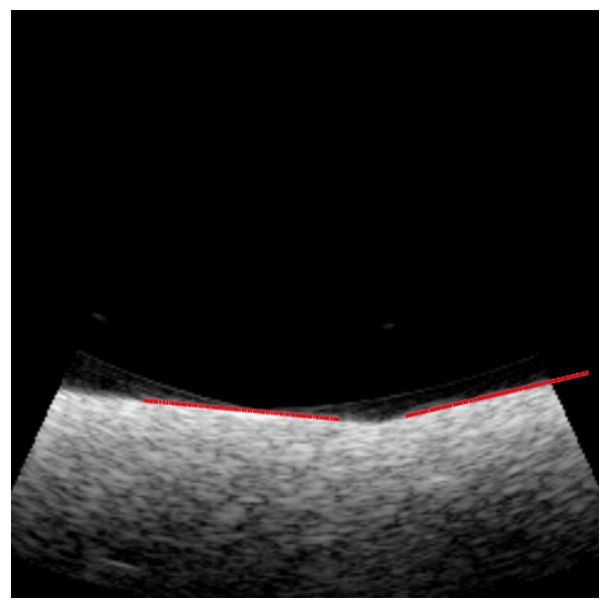

(b) registered planes model

Fig. 3. Planes model registered into 3D ultrasound data. 


\subsection{Model to Ultrasound Registration}

The coarse orientation of the ultrasound probe relative to the calibration phantom is always similar in each calibration measurement because tracking spheres of the probe and the phantom have to show in the direction of the tracking camera. Therefore always the same initial guess for the registration transformation can be used. The registration procedure is very fast (within few seconds) and for three different calibration trials accurate registration results have been reached. An impression of the registration quality is shown in Fig. 3b.

\subsection{Calibration Reproducibility}

Different calibration trials should ideally yield the same calibration transformation. A metric to evaluate the reproducibility is the mean of the distances $\Delta x_{i}=T_{I \leftarrow S}^{k} x_{i}-T_{I \leftarrow S}^{l} x_{i}$ of $n$ points $x_{1}, \ldots, x_{n} \in \mathbb{R}^{3}$ transformed with calibration transformations from different trials $k$ and $l$. We took $3 \times 3 \times 3$ points on a regular grid of size $8^{3} \mathrm{~cm}$ inside the ultrasound volume and investigated three calibration trials with differently rotated probe orientations. Between the first and second trial we measured a mean distance of $1.1( \pm 0.4) \mathrm{mm}$, between the first and third of $0.5( \pm 0.2) \mathrm{mm}$ and between the second and third of $1.5( \pm 0.6)$ $\mathrm{mm}$.

\section{Discussion}

The advantages of the proposed calibration procedure to previous approaches are the low costs of the phantom (material costs below 50 EUR), the reachability of the phantom features allowing the direct measurement of the phantom features with a tracked pointer, the clear images of the features without artifacts enabling an automatic detection of the features and thus a fast and automatic calibration procedure for $3 \mathrm{D}$ ultrasound probes.

\section{References}

1. Gronningsaeter A, Kleven A, Ommedal S, et al. SonoWand, an ultrasound-based neuronavigation system. Neurosurgery. 2000;47(6):1373-9.

2. Beller S, Hünerbein M, Lange T, et al. Image-guided surgery of liver metastases by 3D ultrasound-based optoelectronic navigation. Brit J Surg. 2007;94(7):866-75.

3. Fenster A, Downey D, Cardinal H. Three-dimensional ultrasound imaging. Phys Med Biol. 2001;46:R67-R99.

4. Mercier L, Lango T, Lindseth F, et al. A review of calibration techniques for freehand 3-D ultrasound systems. Ultrasound Med Biol. 2005;31(4):449-71.

5. Hsu P, Prager R, Gee A, et al. Freehand 3D ultrasound calibration: a review. Department of Engineering, University of Cambridge; 2007. 584.

6. Lange T, Eulenstein S. Calibration of swept-volume 3D ultrasound. In: Houston A, Zwiggelaar R, editors. Proc Med Image Understand Anal. vol. 3; 2002. p. 29-32.

7. Poon T, Rohling R. Comparison of calibration methods for spatial tracking of a 3-D ultrasound probe. Ultra Med Biol. 2005;31(8):1095-108. 